\title{
EAl Endorsed Transactions

\section{LRSDSFD: low-rank sparse decomposition and symmetrical frame difference method for moving video foreground-background separation}

\author{
Hongqiao $\mathrm{Gao}^{1, *}$ \\ ${ }^{1}$ Sports Department, Luoyang Polytechnic, Luoyang China
}

\begin{abstract}
In scenes with dynamic background or measurement noise, the low-rank sparse decomposition background modeling algorithm based on kernel norm constraint is easy to separate the moving background or noise as part of the foreground and the foreground at the same time, and it has poor modeling performance for complex background. In order to solve this problem, this paper proposes a low-rank sparse decomposition and symmetrical frame difference method for moving video foreground-background separation. Firstly, low-rank sparse decomposition is used to constrain the background matrix. Secondly, the moving objects in the region of interest (ROI) are extracted by symmetrical frame difference method, and the background image is obtained by block background modeling. Numerical experiments show that compared with other five main algorithms, the proposed algorithm can separate moving objects more accurately in the scene with dynamic background.
\end{abstract}

Keywords: low-rank sparse decomposition, symmetrical frame difference, ROI.

Received on 06 November 2021, accepted on 16 November 2021, published on 16 November 2021

Copyright (C) 2021 Hongqiao Gao et al., licensed to EAI. This is an open access article distributed under the terms of the Creative Commons Attribution license, which permits unlimited use, distribution and reproduction in any medium so long as the original work is properly cited.

doi: 10.4108/eai.16-11-2021.172133

"Corresponding author. Email: $910675024 @ q q . c o m$

\section{Introduction}

Video foreground and background separation has always been the core issue in the field of computer vision. As the basis of intelligent monitoring technology, its research will have a direct impact on applications such as target recognition [1], target detection [2], target tracking [3-4].

At present, the mainstream algorithms for moving video foreground and background separation can be divided into optical flow method [5], frame difference method [6] and background modeling method [7-8]. Low rank sparse decomposition model is one of the background modeling methods. In the framework of compressed sensing theory [9-11], Liu et al. [12] proposed robust principal component analysis (RPCA). It regarded all sequences in the video as a large observation matrix, and each video image was equivalent to a column in the observation matrix.

Since the background part of the video does not change with time and the position of the same position in different frames is the same, the background part can be expressed as a low rank matrix. The change of foreground is dynamic and can be expressed as a sparse matrix. Then a separation method called principal component tracking (PCP) is proposed.

RPCA algorithm is easily disturbed by dynamic background and noise in front background separation, and 
the separation effect is poor. Therefore, it is still a challenging problem to accurately separate the prospects. Zhou et al. [13] proposed the decomposition algorithm "GoDec", which divided the objective function in low rank sparse decomposition into foreground, background and noise, and used bilateral random projection instead of singular value decomposition (SVD) in PCP. It improved the robustness of PCP in complex background and noise. In PCP, the kernel norm is the convex envelope of the rank function. The kernel norm is used to constrain the background matrix. Although the original problem is transformed into a convex optimization problem that is easy to solve, it limits the flexibility of dealing with practical problems. Considering that the larger singular value has a greater impact on the matrix approximation performance, $\mathrm{Gu}$ et al. [14] proposed the weighted kernel norm minimization (WNNM) model. The model would adaptively give different singular values with different weights, which improved the flexibility of the model in dealing with actual scenes. Zhang et al. [15] proposed the truncated kernel norm (TNN) model, which kept the large singular value unchanged and only minimized the small singular value, and also received good recovery effect in some scenarios. Since the kernel norm is only the optimal convex approximation form of the matrix rank function, and the approximation of the non convex form will get better recovery results, Nie et al. [16] proposed the schatten-p norm, which was a non convex minimization form of the rank function, which could suppress the noise generated during measurement. But schatten-p norm also faces the same problem as kernel norm, and cannot deal with different singular values flexibly. Inspired by the weighted kernel norm, Xie et al. [17] proposed the weighted schatten-p norm minimization (WSNM) model, which could deal with different singular values more flexibly and was a more accurate rank function approximation [18].

PCP uses L1 norm constraint for the sparse part. Because L1 norm processes each element of the matrix separately, it ignores the spatial structural relationship when the foreground object moves. This property is obviously different from the local motion and periodic motion of the dynamic background. Therefore, Guyon et al. [19] proposed $l_{2,1}$ norm with block sparsity, emphasized the relationship between the low rank of the background and the sparsity of the foreground, and it better separated the foreground objects. Liu et al. [20] proposed a low rank and structured sparse decomposition (LSD) model. The sparse part was a structured sparse norm based on overlapping groups, which could adapt to complex and changeable video scenes. Inspired by the weighted schatten-p norm in reference [18] and the structured sparse norm in reference [20], a new low rank sparse decomposition model was proposed by combining the symmetrical frame difference with the structured sparse norm, which was solved by the augmented Lagrange multiplier (ALM) method. The experimental results in multiple groups of complex video scenes showed that, the proposed model had a good separation effect on moving targets in dynamic background.

\section{Background image generation based on symmetrical frame difference processing}

A preliminary moving target image is obtained by symmetrical frame difference processing, and then the block background modeling is applied to generate the background image [21-23].

\subsection{Symmetrical frame difference processing}

The input image of the symmetrical frame difference processing is the consecutive three frames of dimensionalreduction grayscale image (frame $\mathrm{i}-1, \mathrm{i}, \mathrm{i}+1$ ), denoted as $I_{i-1}, I_{i}, I_{i+1}$. Symmetrical frame difference processing mainly includes difference processing, median filtering and image binarization.

1) Difference processing

Absolute difference calculation is performed for $I_{i}$ and $I_{i-1}, I_{i}$ and $I_{i+1}$ respectively to obtain difference images $D_{i}^{1}$ and $D_{i}^{2}$. The calculation formula is as follows:

$$
\begin{aligned}
& D_{i}^{1}=\left|I_{i}-I_{i-1}\right| \\
& D_{i}^{2}=\left|I_{i+1}-I_{i}\right|
\end{aligned}
$$

2) Median filtering.

In order to eliminate the difference image noise initially, the median filter operator with $2 \times 2$ pixel size is used to filter $D_{i}^{1}$ and $D_{i}^{2}$.

3) Image binarization.

We take $D_{i}^{1}$ binarization as an example,

$$
B_{i}^{1}(x, y)= \begin{cases}1 & D_{i}^{1}(x, y) \geq T_{i}^{1} \\ 0 & D_{i}^{1}(x, y)<T_{i}^{1}\end{cases}
$$

Where $B_{i}^{1}$ is the binary image of $D_{i}^{1}$, that is, the binary image of the moving target. $D_{i}^{1}(x, y)$ is the pixel value of $(\mathrm{x}, \mathrm{y})$ pixel point in difference image $D_{i}^{1}$. $B_{i}^{1}(x, y)$ is the pixel value of $(\mathrm{x}, \mathrm{y})$ pixel point in binarization image $B_{i}^{1} . T_{i}^{1}$ is the threshold, which is 
related to the OTSU optimal segmentation threshold $t_{i}^{1}$ of $D_{i}^{1}, T_{i}^{1}=t_{i}^{1}-\Delta$. If $B_{i}^{1}(x, y)=1$, then $B_{i}^{1}(x, y)$ is the foreground pixel; otherwise, it is the background pixel. In this case, let $\Delta=25$.

So far, the binarization image $B_{i}^{1}$ of $D_{i}^{1}$ is obtained. Similarly, the binarized image $B_{i}^{2}$ of $D_{i}^{2}$ can be obtained. Binarization image $B_{i}^{1}$ and $B_{i}^{2}$ are shown in figure 1 .

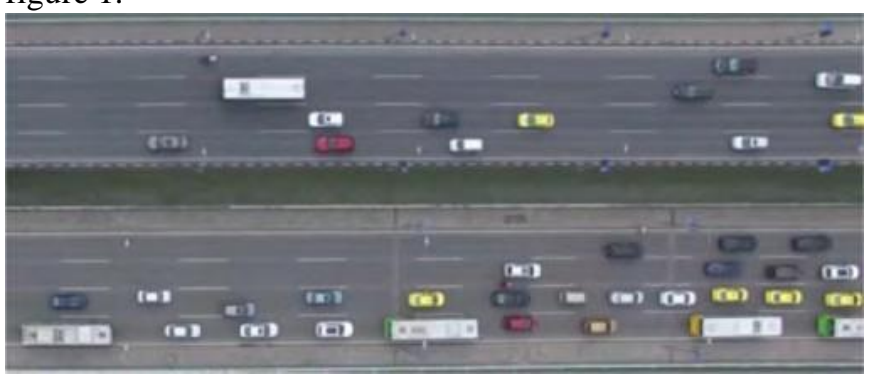

(a) Raw moving image

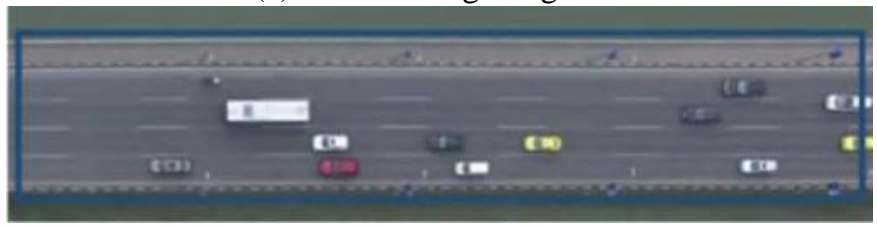

(b) ROI

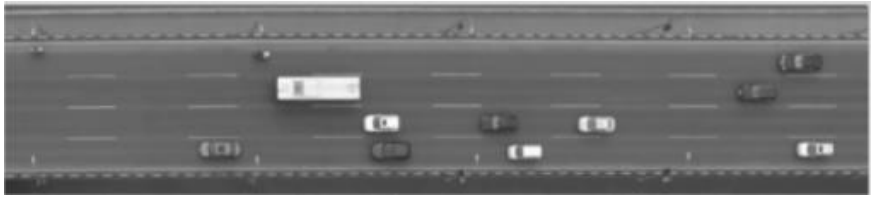

(c) Reduced dimensions grayscale image

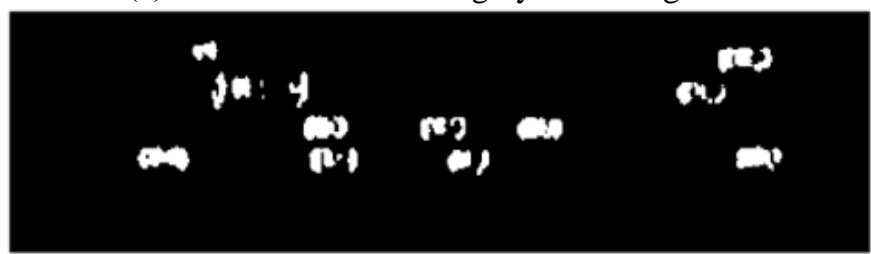

(d) $B_{i}^{1}$

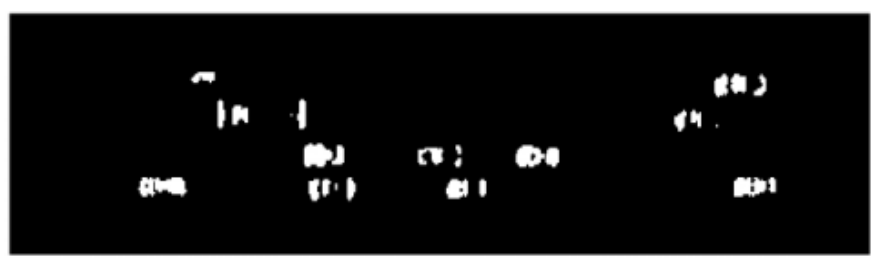

(e) $B_{i}^{2}$

Figure 1. Binary image

\subsection{Preliminary recognition of moving target image}

Image information is fused. OR operation is performed on $B_{i}^{1}$ and $B_{i}^{2}$, and then denoising is performed to form the preliminary recognition result $B_{i}$ of the moving target, as shown in figure 2 . It can be seen that the fusion of the difference results of three frames based on the symmetrical frame difference method can eliminate or reduce the dilated pixels inside the image, enhance the integrity of the target, and effectively improve the detection effect of moving targets.

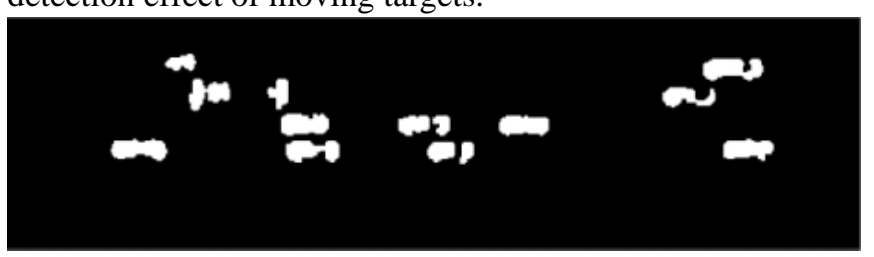

Figure 2. Preliminary recognition results of moving objects

\subsection{Block background modeling}

This paper proposes a block modeling method based on symmetrical frame difference, and the process is shown in figure 3. The main steps are described as follows:

1) The size of the initial background image $I_{b}$ is consistent with the symmetrical frame difference result $B_{i}$ and the dimensional-reduction gray scale image $I_{i}$.

2) Sub-block division. $B_{i}, I_{i}, I_{b}$ are divided into $\mathrm{M} \times \mathrm{N}$ sub-blocks (the size of sub-blocks depends on the actual situation. For example, if $\mathrm{M}=48$ and $\mathrm{N}=27$, the size of sub-blocks is $20 \times 20$ pixels). The pixels of $B_{i}$ are divided into foreground pixels (white pixels represent moving objects) and background pixels (black pixels represent background). Therefore, the sub-block of $B_{i}$ may have the following three situations: background sub-block (all background pixels, sub-block 3 in figure 4), sub-block containing background pixels and foreground pixels (subblock 2 in figure 4), and foreground sub-block (all foreground pixels, sub-block 1 in figure 4).

3) Generating background image $I_{b}$. Background image $I_{b}$ can be successfully generated only when all $\mathrm{M} \times \mathrm{N}$ sub-blocks in $I_{b}$ generate backgrounds. Therefore, background judgment is made on all sub-blocks of $B_{i}$ through $\mathrm{j}++$ loop first. If $I_{b}$ is successfully generated, background modeling is completed. Otherwise, it is necessary to enter the i++ loop to carry out the next round of symmetrical frame difference processing to obtain the 
updated $B_{i}$, and then perform the sub-block background judgment until all $\mathrm{M} \times \mathrm{N}$ sub-blocks in $I_{b}$ generate backgrounds.
Normally, the background modeling process will enter the i++ loop many times, that is, the symmetric frame difference processor will be called many times before the background image can be successfully generated, as shown in figure 5 .

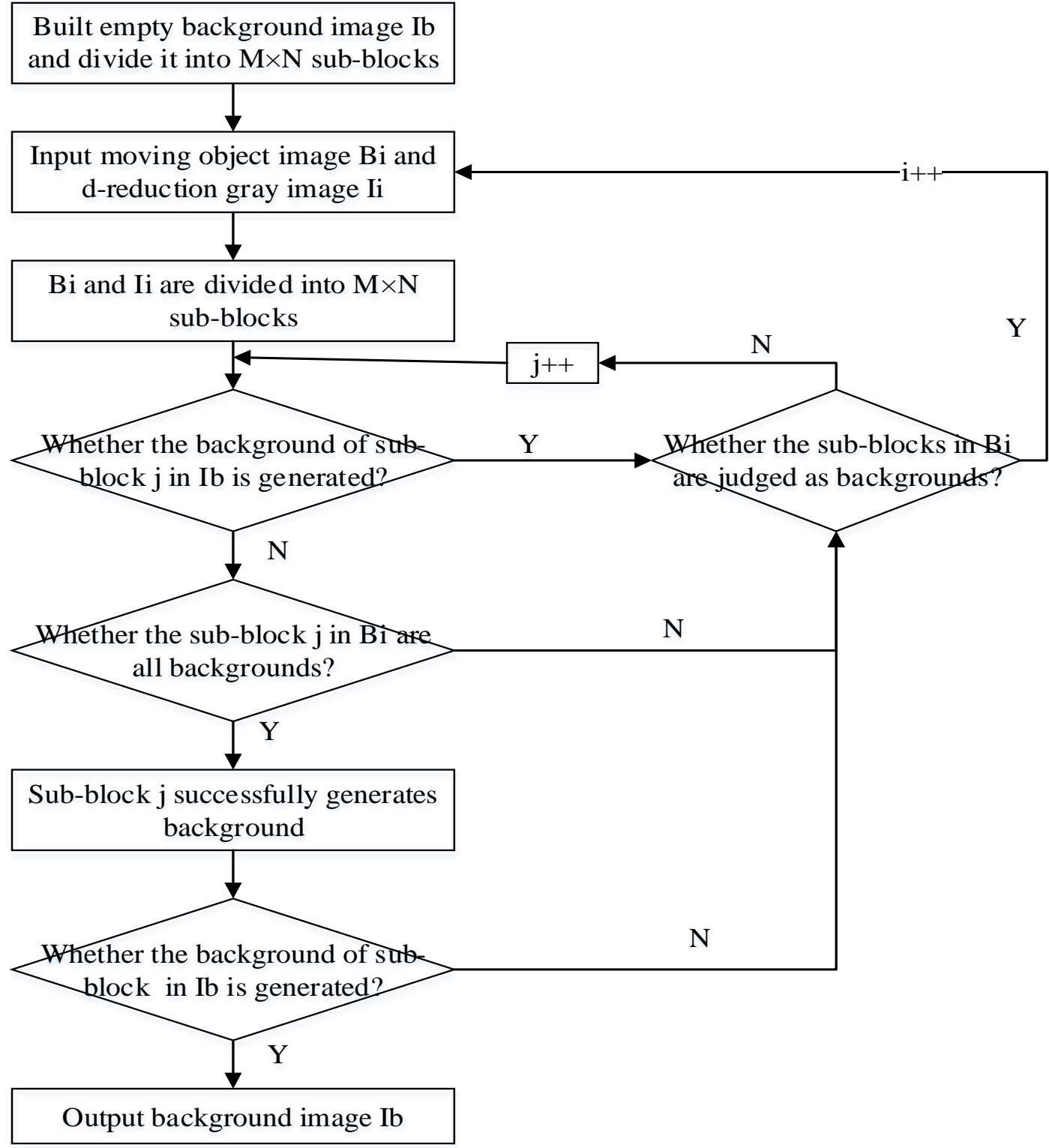

Figure 3. Background Modeling flowchart 


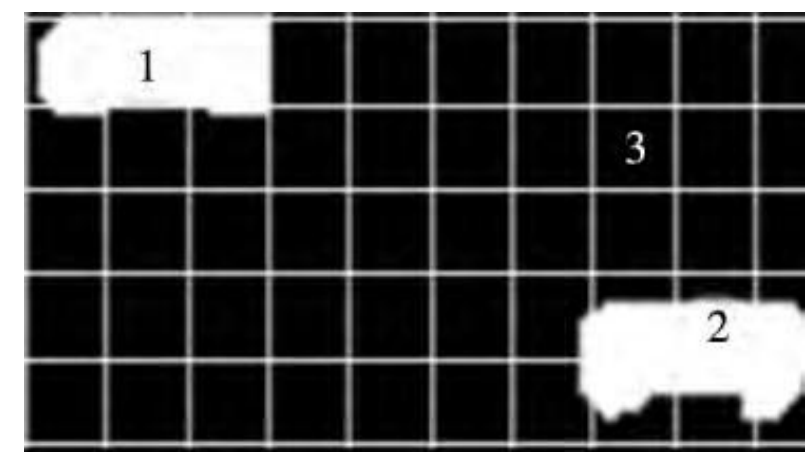

Figure 4. sub-block division of $B_{i}$

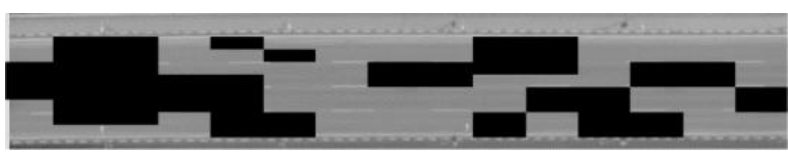

(a) Generating part of background sub-block

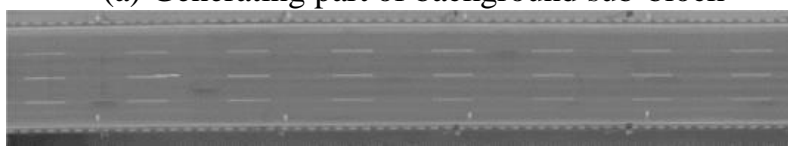

(b) Successfully generated background image

Figure 5. Example of background modeling process

\section{Low rank sparse decomposition model}

The low rank sparse decomposition is to decompose the observation matrix $D \in R^{m \times n}$ of video is decomposed into the sum between low rank matrix $L \in R^{m \times n}$ and sparse matrix $S \in R^{m \times n}$. The mathematical model can be expressed as:

$$
\min _{L, S} R_{\text {rank }}(L)+\lambda\|S\|_{0}, \text { s.t. } D=L+S
$$

Where $R_{\text {rank }}(\cdot)$ represents the rank function of matrix $\mathrm{L}$, that is, the rank size of matrix $\mathrm{L} .\|\cdot\|_{0}$ represents the quasi zero norm of matrix $\mathrm{S}$, that is, the number of nonzero elements in matrix $S . \lambda>0$ is a penalty factor. Because the rank function and quasi-zero norm in equation (4) are non-convex and non-smooth, it is an NP hard problem.

The convex relaxation of equation (4) is transformed into a convex optimization problem, and its mathematical model can be expressed as:

$$
\min _{L, S}\|L\|+\lambda\|S\|_{1}, \text { s.t. } D=L+S
$$

Where $\|\cdot\|_{*}$ represents the kernel norm of matrix $\mathrm{L}$, that is, the sum of singular value of matrix $\mathrm{L} .\|\cdot\|_{1}$ represents the L1 norm of matrix S, that is, the sum of the absolute values of all elements in matrix $\mathrm{S}$.

\subsection{Low rank modeling}

In RPCA algorithm, the low rank matrix $\mathrm{L}$ is constrained by kernel norm, which is not the best approximation of rank function. The weighted schatten-p norm in reference [24] is a more accurate rank function approximation and can suppress the noise generated during measurement. Therefore, the weighted Schatten-p norm is used in this study to carry out the low-rank constraint, and its expression is:

$$
\|L\|_{w, S_{p}}=\left(\sum_{i=1}^{\min \{m, n\}} \omega_{i} \sigma_{i}^{p}\right)^{1 / p}
$$

Where $0<\mathrm{p} \leq 1$ is the weighting parameter. $\sigma_{i}$ represents the $\mathrm{i}$-th singular value of matrix $\mathrm{L}$. $w=\left[\omega_{1}, \cdots, w_{i}, \cdots, w_{\min (m, n)}\right]$ represents the weight matrix of corresponding singular value $\sigma_{i}$, it is a nonnegative vector. $\omega_{i} \geq 0$ satisfies non decrement, i.e. $0 \leq \omega_{1} \leq \cdots \leq \omega_{i} \leq \cdots \leq \omega_{\min (m, n)}$. Then, the weighted schatten-p norm is given to the power of $\mathrm{P}$, and the result is obtained.

$$
\|L\|_{w, S_{p}}^{p}=\sum_{i=1}^{\min \{m, n\}} \omega_{i} \sigma_{i}^{p}
$$

Where, the weight calculation formula $\omega_{i}=C \sqrt{m n} /\left[\sigma_{i}(L)+\varepsilon\right] . \mathrm{C}$ is a constant greater than zero. $\varepsilon$ represents a minimal constant, usually $\varepsilon=10^{-16}$.

\subsection{Sparse modeling}

In RPCA algorithm, the sparse matrix $\mathrm{S}$ is constrained by L1 norm, which processes each element in the matrix independently, but the movement of foreground targets is continuous in space. Using this structural prior information, reference [20] proposed a structured sparse norm based on overlapping groups.

As shown in figure 6, assuming the sparse foreground on the $8 \times 8$ pixel image has two different distributions, as shown in figure 6 (a) and figure 6 (b). In the figure, white indicates that the pixel gray value is large, and black indicates that the pixel gray value is small. Since L1 norm represents the sum of the absolute values of all elements, L1 norm will have similar values in both cases. Structured sparse norm is a $t \times t$ pixel window slides on the image line 
by line and column by column to obtain the $l_{\infty}$ norm corresponding to each window. This paper uses $3 \times 3$ pixel window, six pixels overlap between adjacent windows. On the image with $8 \times 8$ pixel, 36 groups sub-space $g$ of $3 \times 3$ pixel can be obtained in advance, that is, $\mathrm{G}=\{\mathrm{g} 1, \mathrm{~g} 2, \ldots, \mathrm{g} 36\}$. Then it takes the $l_{\infty}$ norm of each subspace, that is, the maximum value of each subspace. Therefore, two significantly different values will be generated. For figure6 (a) and figure6 (b), since there are more groups of pixels with larger values in figure $6(d)$, the value of figure 6 (c) will be much smaller than that of figure $6(\mathrm{~d})$. Under the requirement of minimum sparsity of foreground, figure6 (a) will be more likely to be considered as foreground.
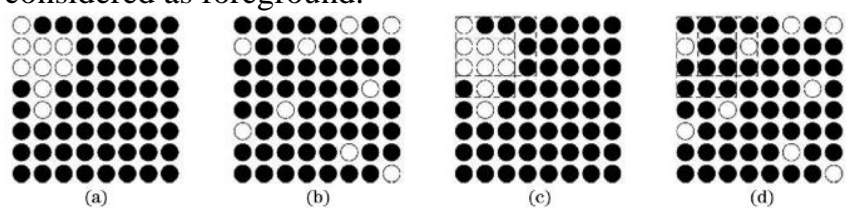

Figure 6. Structured figures based on $3 \times 3$ pixel overlapping group. (a) (b) Two different foreground distributions; (c) (d) overlapping group of two different distributions.

In the foreground matrix, each frame image is regarded as a column of elements, then the structured sparse norm constraint for the whole foreground matrix $\mathrm{S}$ can be expressed as:

$$
\Omega(S)=\sum_{j=1}^{n} \sum_{g \in G}\left\|s_{g}^{(j)}\right\|_{\infty}
$$

Where $s_{g}^{(j)}$ represents the element covered by the window in $\mathrm{j}$-th frame. $s^{(j)} \in R^{m}$ represents the $\mathrm{j}$-th column element of the foreground matrix $S$, that is, the $j$ th frame of the video. $g$ represents the subspace of the elements covered by the window. G represents the set of $g$, and $\left\|s_{g}^{(j)}\right\|$ represents the $l_{\infty}$ norm of $s_{g}^{(j)}$.

\subsection{Model establishment and solution}

Based on the above two norm constraints, the low rank sparse decomposition model is obtained by replacing the low rank part and sparse part in equation (5), which can be expressed as:

$$
\min _{L, S}\|L\|_{w, S_{p}}^{p}+\lambda \Omega(S), \text { s.t. } D=L+S
$$

The above optimization problem is solved by ALM algorithm. The specific solution steps are as follows.

1) The augmented Lagrange function is constructed.

$$
\begin{aligned}
& L(L, S, Y ; \mu)=\|L\|_{w, S_{p}}^{p}+\lambda \Omega(S) \\
& +<Y, D-L-S>+\frac{\mu}{2}\|D-L-S\|_{F}^{2}
\end{aligned}
$$

Where $\mu$ is a positive penalty factor. $\mathrm{Y}$ is the Lagrange multiplier vector. $\|\cdot\|_{F}$ represents the $\mathrm{F}$ norm of the matrix.

2) Solve the following three sub problems.

$$
\begin{aligned}
& L=\underset{L}{\arg \min } L(L, S, Y ; \mu) \\
& S=\underset{S}{\arg \min } L(L, S, Y ; \mu) \\
& Y=Y+\mu(D-L-S)
\end{aligned}
$$

3) Fix S and $Y$ and solve L.

$$
\begin{aligned}
& L_{k+1}=\underset{L}{\arg \min } \frac{1}{\mu_{k}}\|L\|_{w, S_{p}}^{p} \\
& +0.5\left\|L-\left(D-S_{k}+\frac{Y_{k}}{\mu_{k}}\right)\right\|_{F}^{2}
\end{aligned}
$$

The generalized soft threshold algorithm (GST) is used to solve equation (14).

4) Fix L and Y and solve S.

$$
\begin{aligned}
& S_{k+1}=\underset{S}{\arg \min } \frac{\lambda}{\mu_{k}} \Omega(S) \\
& +0.5\left\|D-L_{k+1}+\frac{Y_{k}}{\mu_{k}}-S\right\|_{F}^{2}
\end{aligned}
$$

5) Fix L and $\mathrm{S}$ and solve $\mathrm{Y}$.

$$
Y_{k+1}=Y_{k}+\mu_{k}\left(D-L_{k+1}-S_{k+1}\right)
$$

6) Update $\mu_{k}$.

$$
\mu_{k+1}=\min \left(\rho \mu_{k}, 10^{7} \mu_{k}\right)
$$

\section{Experimental results and analysis}

\subsection{Experimental environment}

In order to verify the effectiveness of the proposed algorithm, nine videos with complex background characteristics in three mainstream databases: I2R [25], CDnet2014 [26] and Wallflower [27] are selected for comparative experiments. I2R: Airport, Bootstrap, Curtain, SwitchLight and WaterSurface; CDnet2014: Highway, Office; Wallflower: CamouFlage, WavingTrees. The characteristics of the selected dataset are shown in Table 1. 
Table 1. Information of 9 video datasets for comparison

\section{Dataset}

Airport

Bootstrap

$160 \times 120$

Curtain

$160 \times 128$

SwitchLight

$160 \times 128$

WaterSurface

$160 \times 128$

Highway

$360 \times 240$

Office

$360 \times 240$

CamouFlage

$160 \times 120$

WavingTrees
$160 \times 120$
Scene feature

Dynamic background, changing time information on the frame

Static background, some moving people in the room

Dynamic background, the curtains moving slowly and light change gradually

Dynamic background, light mutation

Dynamic background, watersurface fluctuation

Dynamic background, the high speed vehicle and the moving shadow

Staticback ground, a people moving locally with strong light

Dynamic background, background with a changing screen

Dynamic background, the swinging branch
100 frames are selected equidistantly for each video data set, and compared with the mainstream GoDec, PCP, LSD, WNNM and WSNM algorithms. The running environment of all experiments in this paper is Intel (R) core (TM) i7-8550U, $1.80 \mathrm{GHz} 8 \mathrm{~GB}$ memory and matlab2017a [28-30]. All the experimental results are processed by median filter.

According to the experience of existing algorithms, the termination condition used in this experiment is $\left\|D-L_{k+1}-S_{k+1}\right\|_{F}^{2} /\|D\|_{F}^{2} \leq 10^{-7}$, and the value of some parameters are: $\varepsilon=10^{-16}, \rho=1.05, \lambda=1 / \sqrt{m}$, $\mu_{1}=12.6 / \sigma_{1}(D), \sigma_{1}(D)$ represents the maximum singular value of matrix D. For the value of parameter $p$, according to the quantitative experiment in literature [18], when $\mathrm{p}=0.7$, the recovery effect is the best and the sensitivity to rank and noise is the smallest. Therefore, $p$ is taken as 0.7 in this paper. Please refer to references $[13,14,15,17,20]$ for the specific experimental parameters of the comparison algorithm. The value of $\mathrm{C}$ will be discussed in 4.2 .

\subsection{Evaluation index}

The comprehensive measurement index F-measure is used to evaluate the separation effect, and its expression is:

$$
F=\frac{2 r p}{r+p}
$$

Where $p=\frac{T P}{T P+F P}$ denotes precision, that is, the ratio of foreground pixels correctly recovered by the algorithm to all foreground pixels recovered by the algorithm. $r=\frac{T P}{T P+F N}$ denotes recall, that is, the ratio of foreground pixels correctly recovered by the algorithm to real foreground pixels. TP indicates that the foreground pixels are judged as foreground pixels, that is, the number of foreground pixels judged correctly by the algorithm. FP indicates that the background pixel is judged as the foreground pixel, that is, the number of foreground pixels judged wrong by the algorithm. FN indicates that the foreground pixels are judged as background pixels, that is, the number of foreground pixels not judged by the algorithm [31]. According to equation (14), the higher the value of F-measure, the better the separation effect.

Taking the airport dataset as an example, other parameters are fixed, the value of $\mathrm{C}$ is between $[0.1,1]$, and the analysis is carried out in steps of 0.1 . As can be seen from figure 7 , when $\mathrm{C}=0.1$, the $\mathrm{F}$-measure value is the highest, so $\mathrm{C}$ in this paper is 0.1 . 


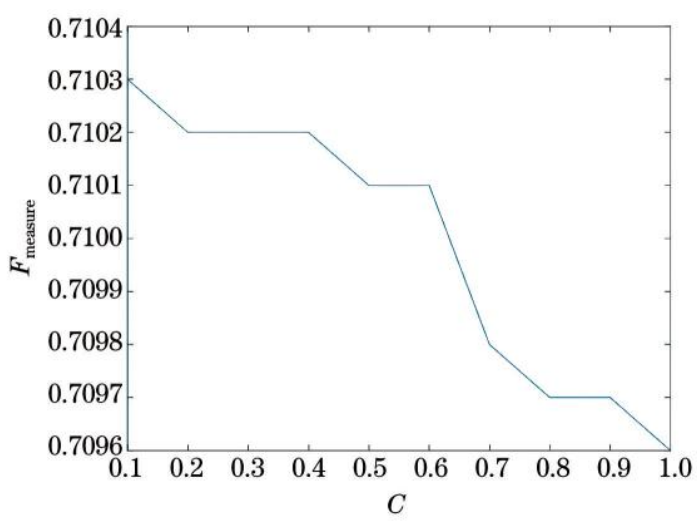

\subsection{Comparison experiments}

The number of real foreground frames provided by different databases also varies. I2R provides 20 frames, CDnet2014 provides all the real foreground frames, and Wallflower only provides the most representative one frame. The experimental results obtained are shown in figure 8 , and $\mathrm{F}$ values under quantitative experiment are given in Table 2 .

Figure 7. F-measure values under the different $\mathrm{C}$ values on Airport dataset.

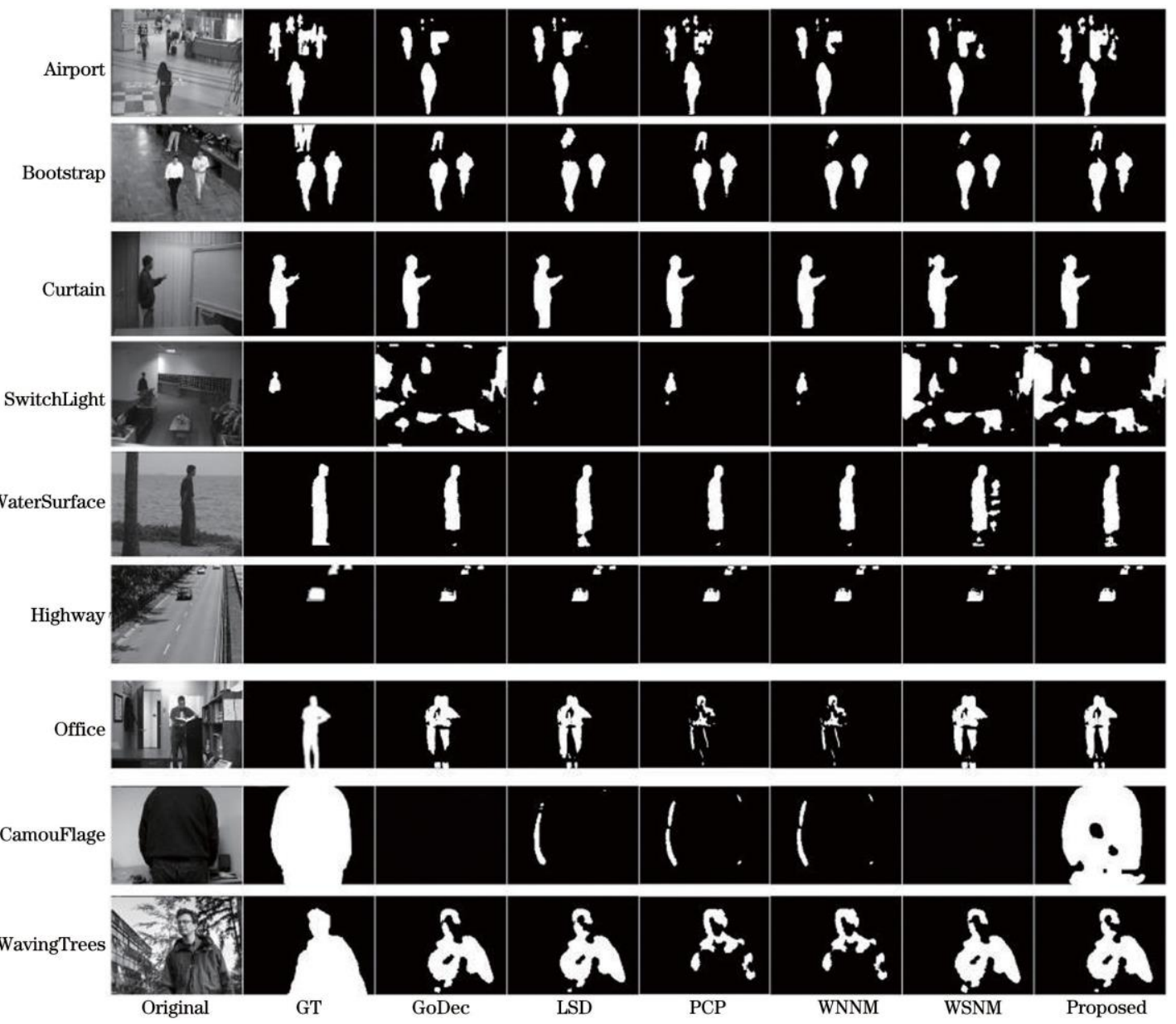

Figure 8. Results of six algorithms on nine sequences 
Table 2. F values with different methods

\begin{tabular}{ccccccc}
\hline video & & \multicolumn{3}{c}{ F-measurement } \\
GoDec & LSD & PCP & WNNM & WSNM & Proposed \\
airport & 0.6712 & $\mathbf{0 . 7 3 8 4}$ & 0.6712 & 0.6712 & 0.6277 & $\underline{0.7214}$ \\
bootstrap & 0.5221 & $\underline{0.5237}$ & 0.5199 & 0.5199 & 0.4977 & $\mathbf{0 . 5 2 4 6}$ \\
curtain & 0.8418 & $\underline{0.8707}$ & 0.8420 & 0.8418 & 0.8381 & $\mathbf{0 . 8 7 2 5}$ \\
switchlight & 0.1379 & 0.7995 & $\underline{0.8077}$ & $\mathbf{0 . 8 1 0 0}$ & 0.1401 & 0.1433 \\
watersurface & 0.8850 & $\mathbf{0 . 9 2 8 1}$ & 0.8788 & 0.8772 & 0.8523 & $\underline{0.9207}$ \\
highway & 0.8163 & 0.8973 & $\underline{0.8998}$ & $\mathbf{0 . 9 0 0 5}$ & 0.8254 & 0.8980 \\
office & 0.5024 & 0.4758 & 0.3888 & 0.3903 & 0.5054 & 0.4759 \\
camouflage & 01221 & 0.1767 & $\underline{0.2264}$ & 0.2259 & 0.0478 & $\mathbf{0 . 9 5 9 8}$ \\
wavingtrees & 0.7168 & $\mathbf{0 . 7 3 2 4}$ & 0.4240 & 0.4257 & 0.7192 & $\underline{0.7319}$ \\
average & 0.5672 & $\underline{0.6726}$ & 0.6176 & 0.6180 & 0.5574 & $\mathbf{0 . 6 9 4 2}$
\end{tabular}

Figure 8 shows the foreground effects of GoDec, LSD, PCP, WNNM, WSNM and the proposed in this paper in nine scenarios (airport, bootstrap, curtain, switchlight, watersurface, highway, office, camouflage and wavingtrees). It can be seen from the bootstrap data set that the proposed algorithm is not much different from other methods for front background separation under static background. It can be seen from the switchlight dataset that the method in this paper cannot be applied to the case of sudden illumination change, but it has excellent separation effect under other complex and changeable dynamic backgrounds. Especially on the camouflage dataset, its background is a changing computer screen [32-34]. When other algorithms fail, the proposed algorithm can still correctly separate the foreground and background, It shows the excellent performance. From the measurement index $F$ values in Table 2, it can be seen that most $F$ values with dynamic background in the nine data sets belong to the highest value or the second highest value, and the average $F$ value of the proposed in this paper is the highest in all methods [35-40]. Therefore, in a comprehensive view, the performance of new method is better than other comparison algorithms on the whole.

\section{Conclusion}

Based on the above two constraints, the low rank and sparse parts are modeled respectively, and a low rank sparse decomposition model for video foreground and background separation is proposed. The low rank part adopts the symmetrical frame difference method, which can more accurately approximate the rank function, suppress the noise generated during measurement. It is more suitable for background modeling. The sparse part adopts the structured sparse norm based on overlapping groups, and uses the foreground structure information to judge the foreground target more accurately, which is more conducive to foreground modeling. Experimental results show that the proposed algorithm is not robust to sudden illumination scenes, but it can obtain good separation effect in static background and better separation effect in complex and diverse dynamic background. In the next work, not only the sudden change of illumination should be considered to make it more robust, but also the movement of camera should be considered.

\section{Acknowledgements.}

The author would like to thank the reviewers and the editor for their valuable comments

\section{References}

[1] Shoulin Yin, Ye Zhang, Shahid Karim. Large Scale Remote Sensing Image Segmentation Based on Fuzzy Region Competition and Gaussian Mixture Model[J]. IEEE Access. volume 6, pp: 26069 - 26080, 2018.

[2] Yin Shoulin, Liu Jie, Teng Lin. A new krill herd algorithm based on SVM method for road feature extraction[J]. Journal of Information Hiding and Multimedia Signal Processing, v 9, n 4, p 997-1005, July 2018. 
[3] Yin K, Li J, Li L, et al. Adaptive Feature Update ObjectTracking Algorithm in Complex Situations[J]. Acta Optica Sinica, 2019, 39(11):1115002.

[4] Q. Wang, Siyuan, et al. Approach for recognizing and tracking beacon in inter-satellite optical communication based on optical flow method.[J]. Optics express, 2018, 26(21):28080-28090.

[5] Shoulin Yin, Ye Zhang. Singular value decompositionbased anisotropic diffusion for fusion of infrared and visible images[J]. International Journal of Image and Data Fusion, 10(2), pp: 146-163, 2019.

[6] Peng B, Cai X, Zhang Y, et al. Automatic vehicle detection from UAV videos based on symmetrical frame difference and background block modeling[J]. Journal of Southeast University(Natural Science Edition), 47(4):685-690, 2017.

[7] Elkabetz A, Yitzhaky Y. Background modeling for moving object detection in long-distance imaging through turbulent medium[J]. Applied Optics, 2014, 53(6):1132.

[8] Zhu X, Zhang C, Xue J P, et al. Background subtraction via time continuity and texture consistency constraints[J]. Journal of the Optical Society of America A, 2019, 36(9):1495.

[9] Boyer C, Bigot J, Weiss P. Compressed sensing with structured sparsity and structured acquisition[J]. Applied and Computational Harmonic Analysis, 2019, 46(2):312350 .

[10] D Sjoerd, Christian J H, Holger R. One-bit compressed sensing with partial Gaussian circulant matrices[J]. Information and Inference: A Journal of the IMA, 2019(3):3.

[11] S. Daei, F. Haddadi, A. Amini and M. Lotz, "On the Error in Phase Transition Computations for Compressed Sensing," in IEEE Transactions on Information Theory, vol. 65, no. 10 , pp. 6620-6632, Oct. 2019, doi: 10.1109/TIT.2019.2920640.

[12] Liu S S, Ying G G, Liu S, et al. Analysis of 21 progestagens in various matrices by ultra-highperformance liquid chromatography tandem mass spectrometry (UHPLC-MS/MS) with diverse sample pretreatment[J]. Analytical \& Bioanalytical Chemistry, 2014, 406(28):7299-7311.

[13] Zhou, T., Tao, D.: GoDec: Randomized low-rank \& sparse matrix decomposition in noisy case. In: International Conference on Machine Learning, pp. 33-40 (2011)

[14] Gu S, Lei Z, Zuo W, et al. Weighted Nuclear Norm Minimization with Application to Image Denoising[C]// 2014 IEEE Conference on Computer Vision and Pattern Recognition (CVPR). IEEE, 2014.

[15] D. Zhang, Y. Hu, J. Ye, X. Li and X. He, "Matrix completion by Truncated Nuclear Norm Regularization," 2012 IEEE Conference on Computer Vision and Pattern
Recognition, 2012, pp. 2192-2199, doi: 10.1109/CVPR.2012.6247927.

[16] F. Nie, H. Wang, X. Cai, H. Huang and C. Ding, "Robust Matrix Completion via Joint Schatten p-Norm and lpNorm Minimization," 2012 IEEE 12th International Conference on Data Mining, 2012, pp. 566-574, doi: 10.1109/ICDM.2012.160.

[17] Y. Xie, S. Gu, Y. Liu, W. Zuo, W. Zhang and L. Zhang, "Weighted Schatten \$p\$ -Norm Minimization for Image Denoising and Background Subtraction," in IEEE Transactions on Image Processing, vol. 25, no. 10, pp. 4842-4857, Oct. 2016, doi: 10.1109/TIP.2016.2599290.

[18] Teng Lin, Hang Li and Shoulin Yin. Modified Pyramid Dual Tree Direction Filter-based Image De-noising via Curvature Scale and Non-local mean multi-Grade remnant multi-Grade Remnant Filter [J]. International Journal of Communication Systems. v 31, n 16, November 10,pp. e.3486.1-e.3486.12, 2018.

[19] C. Guyon, T. Bouwmans and E. Zahzah, "Foreground detection based on low-rank and block-sparse matrix decomposition," 2012 19th IEEE International Conference on Image Processing, 2012, pp. 1225-1228, doi: 10.1109/ICIP.2012.6467087.

[20] Liu X, Zhao G, Yao J, et al. Background Subtraction Based on Low-Rank and Structured Sparse Decomposition.[C]// IEEE International Conference on Electronics. IEEE, 2013.

[21] Shi F, Qiu F, X Li, et al. Detecting and Tracking Moving Airplanes from Space Based on Normalized Frame Difference Labeling and Improved Similarity Measures[J]. Remote Sensing, 2020, 12(21):3589.

[22] Wang S, F Yu, Zhou C, et al. Straw Burning Detection Method Based on Improved Frame Difference Method and Deep Learning[C]// 2020 IEEE 5th International Conference on Image, Vision and Computing (ICIVC). IEEE, 2020.

[23] Nan Zhao, Xiao-Wei Wang, and Shou-Lin Yin. Research of Fire Smoke Detection Algorithm Based on Video [J]. International Journal of Electronics and Information Engineering. Vol. 13, No. 1, pp. 1-9, 2021.

[24] Zha Z, Zhang X, Wu Y, et al. Non-Convex Weighted Schatten p-Norm Minimization based ADMM Framework for Image Restoration. 2017. arXiv:1704.07056

[25] Liyuan Li, Weimin Huang, Irene Yu-Hua Gu and Qi Tian, "Statistical modeling of complex backgrounds for foreground object detection," in IEEE Transactions on Image Processing, vol. 13, no. 11, pp. 1459-1472, Nov. 2004, doi: 10.1109/TIP.2004.836169.

[26] Wang Y, Jodoin P M, Porikli F, et al. CDnet 2014:an expanded change detection benchmark dataset [C]//2014 IEEE Conference on Computer Vision and Pattern Recognition Workshops, June 23-28, 2014, Columbus, OH, USA. New York: IEEE Press,2014: 393-400. 
[27] Yufeng Wei, Mingli Jing, Lan Li, et al. Video ForegroundBackground Separation via Weighted Schatten-p Normand Structured Sparsity Decomposition[J]. Laser \& Optoelectronics Progress, 58(8), pp.0815008, 2021.

[28] Shoulin Yin, Hang Li, Asif Ali Laghari, et al. A Bagging Strategy-Based Kernel Extreme Learning Machine for Complex Network Intrusion Detection[J]. EAI Endorsed Transactions on Scalable Information Systems. 21(33), e8, 2021. http://dx.doi.org/10.4108/eai.6-10-2021.171247

[29] Qingwu Shi, Shoulin Yin, Kun Wang, et al. Multichannel convolutional neural network-based fuzzy active contour model for medical image segmentation. Evolving Systems (2021). https://doi.org/10.1007/s12530-02109392-3

[30] S. Yin and H. Li. Hot Region Selection Based on Selective Search and Modified Fuzzy C-Means in Remote Sensing Images[J]. IEEE Journal of Selected Topics in Applied Earth Observations and Remote Sensing, vol. 13, pp. 58625871, 2020, doi: 10.1109/JSTARS.2020.3025582.

[31] Shoulin Yin, Hang Li, Desheng Liu and Shahid Karim. Active Contour Modal Based on Density-oriented BIRCH Clustering Method for Medical Image Segmentation [J]. Multimedia Tools and Applications. Vol. 79, pp. 3104931068, 2020

[32] Luong H V, Joukovsky B, Eldar Y C, et al. A DeepUnfolded Reference-Based RPCA Network For Video Foreground-Background Separation[C]// $2020 \quad$ 28th European Signal Processing Conference (EUSIPCO). 2021.

[33] Yang Z, Fan L, Yang Y, et al. Generalized nuclear norm and Laplacian scale mixture based low-rank and sparse decomposition for video foreground-background separation[J]. Signal processing, 2020, 172(Jul.):107527.1107527.10

[34] Yin, S., Li, H. \& Teng, L. Airport Detection Based on Improved Faster RCNN in Large Scale Remote Sensing Images [J]. Sensing and Imaging, vol. 21, 2020. https://doi.org/10.1007/s11220-020-00314-2

[35] Laghari, A.A., Wu, K., Laghari, R.A. et al. A Review and State of Art of Internet of Things (IoT). Arch Computat Methods Eng (2021). https://doi.org/10.1007/s11831-02109622-6

[36] Laghari A A, Laghari M A. Quality of experience assessment of calling services in social network[J]. ICT Express, 2021(2).

[37] Laghari A A, Laghari K, Memon K A, et al. Quality of Experience (QoE) Assessment of Games on workstations and Mobile[J]. Entertainment Computing, 2020, 34:100362.

[38] A. A. Laghari, H. He, A. Khan, N. Kumar and R. Kharel, "Quality of Experience Framework for Cloud Computing (QoC)," in IEEE Access, vol. 6, pp. 64876-64890, 2018, doi: 10.1109/ACCESS.2018.2865967.
[39] Laghari, A.A., He, H., Shafiq, M. et al. Application of Quality of Experience in Networked Services: Review, Trend \& Perspectives. Syst Pract Action Res 32, 501-519 (2019). https://doi.org/10.1007/s11213-018-9471-x

[40] Laghari, A.A., Jumani, A.K. \& Laghari, R.A. Review and State of Art of Fog Computing. Arch Computat Methods Eng 28, 3631-3643, 2021. 